\title{
INDICADORES DAS CONDIÇÕES NUTRICIONAIS NA REGIÃO DO POLONOROESTE. VII. ESTUDO DA PREVALÊNCIA DE DESNUTRIÇÃO E DIARRÉIAS, 1985 (1)
}

\author{
M.H.G.G. da SILVA (2), G.V. da VEIGA (2) \& M.S. AMICUCCI (3)
}

\begin{abstract}
RESUMO
Os autores realizaram um estudo do estado nutricional por antropometria num grupo de 405 crianças de 3 a 72 meses de idade, residentes na área urbana da cidade de Cáceres - Região do Polonoroeste no Estado do Mato Grosso. Mediante a aplicação de um questionário ao responsável pela criança, pesquisou-se a ocorrência de diarréias na mesma população. O objetivo do estudo foi verificar a prevalência de desnutrição e a freqüência de diarréias nessas crianças. Utilizaram-se as classificações propostas por GOMEZ e WATERLOW para verificar a prevalência e a natureza da desnutrição. Foi verificado que a desnutrição ocorreu em $33 \%$ das crianças segundo a classificação de GOMEZ. Segundo os critérios de WATERLOW a forma de desnutrição de maior proporção encontrada foi a pregressa, com percentual de $36 \%$. Entre as crianças desnutridas a frequiência de diarréias foi de $47 \%$ sendo a relação entre diarréia e desnutrição estatisticamente significante.
\end{abstract}

UNITERMOS: Desnutrição; Diarréia.

\section{INTRODUÇÃO}

As carências nutricionais em suas mais variadas formas constituem um dos mais sérios problemas de saúde no mundo.

No Brasil alguns estudos regionais procuram demonstrar a freqüência da desnutrição e a gravidade das suas manifestações e o comprometimento físico e mental dependente de sua instalação na infância etapa vital para o desenvolvimento $1,4,5,15,17,20$.

A desnutrição é definida pela Organização Mundial de Saúde como um "espectro de condições patológicas que provém da simultânea falta, em várias proporções, de proteínas e ca- lorias, ocorrendo mais freqüentemente em préescolares sendo comumente associada a infecções ${ }^{20}$.

A interrelação observada entre desnutrição e infecções parece explicar-se mediante a potencialização de ações: a desnutrição depauperando as defesas imunológicas do hospedeiro, facilita a instalação de quadros infecciosos; estes por outro lado, manifestando-se freqüentemente, comprometem mais ainda o estado nutricional $8.11,18.19,21$.

Dentre os processos infecciosos envolvidos nessa açâo sinérgica com a desnutrição

(1) Auxilio CNPq Processo 141166/MP.

(2) Professor Auxiliar do Departamento de Nutrição - lUFMT.

(3) Professor Assistente do Departamento de Nutrição - FUFMT.

Endereço para correspondência: Universidade Federal de Mato Cirosso - Av. Fernando Corrêa, s/n:" - 78100 Cuiabá, Mato Grosso, Brasil. 


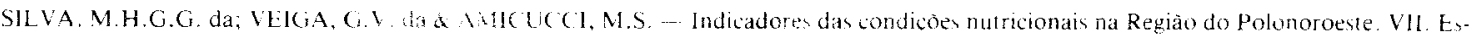

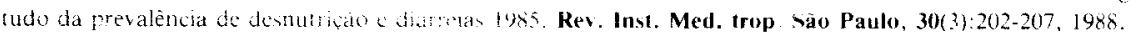

destacam-se o sarampo, as infecçōes respiratorias agudas e as diarréias.

As diarréias figuram como causas muito importantes de morbidade e mortalidade na infância, sendo que a Organização Panamericana de Saúde traça um quadro comparativo da mortalidade infantil por diarréias, onde se verifica que os indices brasileiros $(1.023$ crianças 100 mil) são muito elevados em relação aos de outros países como o Canadá (3/100 mil) e Estados Unidos $(20 / 100 \text { mil })^{19}$.

ATALAB e cols. - 1982, estudando criancas menores de 2 anos no Chile, concluíram que à medida em que deteriora o estado nutricional, ocorre um aumento nos índices de hospitalizaçào por diarréia.

No Brasil, principalmente no Sudeste e Nordeste, há trabalhos objetivando verificar a prevalência da desnutrição em crianças e sua re-. lação com algumas enfermidades infecciosas ${ }^{7}$ 23. 24. 25. Entretanto, não há referências sobre a existência de pesquisas nessa linha na região Centro-Oeste. Este estudo foi realizado com o objetivo de verificar a prevalência da desnutriçào e das diarréias em crianças de 3 a 72 meses de idade, na cidade de Cáceres, região do Polonoroeste no Estado de Mato Grosso.

A cidade de Cáceres situa-se na região noroeste de Mato Grosso e constitui-se num dos municípios que mais contribuíram para a formação histórica do Estado. Encontra-se inserida na área compreendida pelo pantanal matogrossense, próximo à fronteira com a Bolivia e distingue-se por suas características sócio-econômicas, situação geográfica e por pertencer à região em que se desenvolve u Programa de Desenvolvimento Rural Integrado (PDRI) no Estado de Mato Grosso.

Tendo em vista a existência de um programa denominado Diagnóstico em Saúde, desenvolvido na Universidade Federal de Mato Grosso em conjunto com a Secretaria do Estado da Saúde e que se integra ao Projeto Polonoroeste e, considerando que um grupo de docentes do Departamento d? Nutrição da referida Universidade vinha desenvolvendo estudos antropométricos nesta população, optou-se por pesquisar a prevalência de enfermidades infecciosas na mesma população.

\section{MATERIAL E MÉTODOS}

A população de estudo foi composta por
405 crianças residentes na área urbana da cidade de Cáceres - Mato Grosso, incluídas na faixa téria de 3 a 72 meses de idade.

$O$ desenho amostral correspondeu a $100 \%$ dos domicílios sorteados para a entrevista domiciliar (Diagnóstico de Saúde e Epidemiologia das Principais Doenças na Região do Polono: roeste - Estado de Mato Grosso), sendo a população selecionada por sorteio, casual simples ${ }^{14}$.

A avaliação do estado nutricional das crianças foi feita através da antropometria por se constituir em um método amplamente utilizado para tal finalidade $6,9,16,23$. O exame antropométrico obedeceu às recomendações de JELLIFFE $^{13}$ com adaptação do Departamento de Nutrição da Faculdade de Saúde Pública da Universidade de São Paulo. Utilizou-se como padrão de referência o "National Center Health Statistic', (NCHS), recomendado pela Organização Mundial da Saúde'².

A prevalência da desnutrição foi dada pela classificação de GOMEZ10. Para se proceder à avaliação da frequência da infecção (diarréia) nas crianças estudadas, trabalhou-se com as crianças reais desnutridas. Considerou-se como reais desnutridas as crianças classificadas como desnutridas inicialmente pela classificação de GOMEZ e posteriormente também pela classificação de WATERLOW ${ }^{27}$. Para efeitos de análise quando se avalia a freqüência de diarréias nessas crianças a amostra passa a ser de 320 e nào mais de 405 crianças.

O levantamento da ocorrência de diarréias foi feito mediante a aplicação de um formulário em forma de questionário fechado dirigido à mãe ou à pessoa responsável no momento da entrevista, considerando-se como verdadeira a informação de ocorrência da enfermidade referida pelo responsável.

O levantamento dos dados antropométricos e a pesquisa de ocorrência de diarréias foram realizados na mesma data, na Unidade de Apoio à Pesquisa.

Após a coleta dos dados antropométricos as crianças e responsáveis eram encaminhados a entrevistador, devidamente treinado, que procedia ao preenchimento do formulário sobre a ocorrência da enfermidade referida.

As perguntas endereçadas ao responsável pela criança foram: a criança está com diarréia no momento? Se afirmativo: há quantos dias? (Considerou-se um período igual ou maior que 3 dias de presença da doença). Quantas evacua- 
SILVA, M.H.G.G. da; VEIGA, G.V. da \& AMICUCC., Mi.S. - Indicadores das condiçōes nutricionais na Região do Polonoroesle. VII Es-

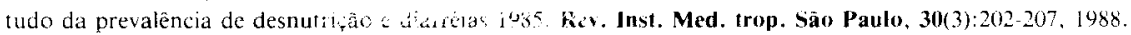

çōes ao dia? (Foi considerado um número igual ou maior que 3 vezes). Caracteristicas das fezes: liquidas? Odor fétido? Nos últimos 30 dias teve diarréia? Quantas vezes?

Foi pesquisada ainda a presença de alguns sinais e sintomas indicarivos de maior gravidade como a presença de febre, perda de apetite, emagrecimento, vômitos, e internaçào.

\section{RESULTADOS}

A Tabela 1 mostra a distribuição das crianças eutróficas e desnutridas, segundo a classificação de GOMEZ, distribuidas por faixa etária, onde se observa que mais de $30 \%$ das crianças estudadas encontravam-se desnutridas.

De acordo com os critérios de WATERLOW a forma de desnutrição de maior prevalência no grupo de crianças estudadas foi a desnutrição pregressa $(36 \%)$, indicando o comprometimento da estatura e da relação peso/altura.
A Tabela 3 descreve a distribuição global das crianças a partir das medidas obtidas, comparando-se com o padrão de referência e classificadas nas diferentes categorias de percentis. Observa-se o dobro do valor esperado $20,9 \%$ no percentil 10 e em relação ao percentil $50,70,6 \%$ das crianças examinadas apresentaram peso inferior ao mesmo.

Em relação ao indicador altura/idade, $80 \%$ da amostra apresentou-se abaixo do percentil 50. Na relação peso/altura $45 \%$ das crianças examinadas encontram-se abaixo do percentil 50.

$\mathrm{Na}$ Tabela 4 observa-se a distribuiçào da ocorrência de diarréia nas crianças consideradas desnutridas e eutróficas neste estudo, onde se observa que mais de $40 \%$ das crianças desnu1 ridas apresentaram a enfermidade, sendo constatado significância ao aplicar o Teste do $\mathrm{X}^{2}$ e $\mathrm{p}$ $<-0.05$.

TABELA 1

Distribuição percentual das crianças de 3 a 72 meses de idade, por faixa etária, segundo a classificação de Gomez (P/I) - Cáceres, Mato Grosso, 1985.

\begin{tabular}{rcccc}
\hline Faixa Etária & Total & $\begin{array}{c}\text { Eutrófilo } \\
\%\end{array}$ & $\begin{array}{c}\text { Desnutrição } \\
\text { GRAU I } \%\end{array}$ & $\begin{array}{c}\text { Desnutrição } \\
\text { GRAU II } \%\end{array}$ \\
\hline $3-12$ & 62 & 85.5 & 14,5 & 0,0 \\
$13-24$ & 62 & 58,1 & 33,8 & 8,1 \\
$25-36$ & 69 & 68,1 & 27,5 & 4,4 \\
$37-48$ & 78 & 66,5 & 34,7 & 3,8 \\
$49-60$ & 66 & 63,6 & 31,9 & 4,5 \\
$61-72$ & 68 & 67,6 & 29,5 & 2,9 \\
\hline TOTAL & 405 & 67,0 & 29,0 & 4,0 \\
\hline
\end{tabular}

Inquérito Nutricional Polonoroeste, 1985.

TABELA 2

Distribuição percentual das crianças de 3 a 72 meses de idade, por faixa etária, segundo a classificação de Waterlow (A/I e P/A) - Cáceres - Mato Grosso, 1985.

\begin{tabular}{rcccc}
\hline Faixa Etária & Eutrófico & $\begin{array}{c}\text { Desnutrição } \\
\text { Pegressa \% }\end{array}$ & $\begin{array}{c}\text { Desnutrição } \\
\text { Atual \% }\end{array}$ & $\begin{array}{c}\text { Desnu trição } \\
\text { Crônica \% }\end{array}$ \\
\hline $3-12$ & 58,1 & 38,7 & 3,2 & 12,9 \\
$13-14$ & 46,8 & 40,3 & - & 1,4 \\
$25-36$ & 65,2 & 32,0 & 1,4 & 5,0 \\
$37-48$ & 61,6 & 30.8 & 2,6 & 4,5 \\
$49-60$ & 50,0 & 41,0 & 4,5 & 2,4 \\
$61-72$ & 63,2 & 32,4 & 1,5 & 4,5 \\
\hline TOTAL & 57,7 & 35,6 & 2,2 & \\
\hline
\end{tabular}

Inquérito Nutricional Polonoroeste, 1985. 
SILVA, M.H.G.G. da; VEIGA, G.V. da \& AMICUCCI, M.S. - Indicadores das condições nutricionais na Região do Polonoroeste. VII. Estudo da prevalência de desnutriçào e diarreias 1985. Rev. Inst. Med. Irop. São Paulo, 30(3):202 207, 1988.

TABELA 3

Distribuição percentual das crianças de 3 a 72 meses de idade por intervalo de percentis, segundo a relação peso/idade, altura/idade e peso/altura - Cáceres, Ma to Grosso, 1985.

\begin{tabular}{|c|c|c|c|c|c|c|}
\hline Percentil & $\mathrm{N}$ & $\begin{array}{c}\text { Peso/Idade } \\
\%\end{array}$ & $\mathrm{~N}$ & $\underset{\%}{\text { Altura/Idade }}$ & $\mathrm{N}$ & $\begin{array}{c}\text { Peso/Altur } \\
\%\end{array}$ \\
\hline$>3$ & 40 & 9,8 & 74 & 18,3 & 05 & 1,2 \\
\hline $3-10$ & 45 & 11,1 & 72 & 17,8 & 16 & 3,9 \\
\hline $19-20$ & 61 & 15,1 & 62 & 15,3 & 32 & 7,9 \\
\hline $20-50$ & 140 & 34,6 & 115 & 28,4 & 129 & 31,9 \\
\hline $50-70$ & 67 & 16,5 & 34 & 8,4 & 111 & 27,4 \\
\hline $70-90$ & 34 & 8,4 & 37 & 9,1 & 81 & 20,0 \\
\hline$>90$ & 18 & 4,5 & 11 & 2,7 & 31 & 7,7 \\
\hline TOTAL & 405 & & 405 & & 405 & \\
\hline
\end{tabular}

Inquérito Nutricional Polonoroeste, 1985.

TABELA 4

Distribuição das crianças de 3 a 72 meses de idade, segundo o estado nutricional e ocorrêncí de diarréias Cáceres, Mato Grosso, 1985.

\begin{tabular}{lrrrr}
\hline \multirow{2}{*}{ Estado Nutricional } & \multicolumn{2}{c}{ Diarréia } & & \\
\cline { 2 - 4 } & Sim & Não & & Total \\
\hline Desnutrido & 52 & 57 & 47.7 & 109 \\
Eutrófico & 70 & 141 & 33.2 & 211 \\
\hline TOTAL & 122 & 198 & 80.9 & 320 \\
\hline
\end{tabular}

$\mathrm{X}^{2}$ observado $=6.433 *$

$\mathrm{X}^{2}$ crítico $(2 \mathrm{gl} ; 5 \%)=3,99$

Inquérito Nutricional Polonoroeste, 1985

\section{DISCUSSÃo}

A avaliação antropométrica e do estado nutricional das crianças incluidas neste estudo mostra uma prevalência de desnutrição de $33 \%$, segundo a classificação de GOMEZ Observa-se também uma alta proporção de desnutridos pregressos, segundo os critérios de WATERLOW: $36 \%$.

Quando se comparam os dados obtidos neste estudo com os de outros trabalhos $3,16,18,26$, observa-se que estes resultados são semelhantes aos de outras populaçōes, como as de São Paulo e Rio de Janeiro.

Compreendendo-se o estado nutricional como a situação orgânica resultante da ingestão, assimilação e utilização dos alimentos, o que encontra-se intimamente relacionado tanto com o meio ambiente como com o meio interno, depreende-se que a interação desarmônica dos dois meios pode determinar a menor ou maior atuação dos fatores interferentes do estado nutricional, como os agentes infecciosos, parasitários e toxicológicos².

Nos estudos de TEIXEIRA ${ }^{25}$, no Pará, abrangendo um total de 116 crianças de até 3 anos de idade foi observado que o grupo de crianças classificadas como desnutridas pelos critérios de Gomez, Marcondes e Ariza Macias, foi acometido de maior número de episódios de doenças. O autor discute no seu trabalho os mecanismos de interação entre a desnutrição e os processos infecciosos, reconhecendo ser a desnutrição um processo de multicasualidade, sendo que os processos infecciosos parecem exercer um papel de grande importância no desenvolvimento das deficiências nutricionais.

A interrelação entre estado nutricional, imunidade e infecção parece constituir-se num elo amplamente reconhecido 1.22. No nosso estudo, a freqüência de diarréias foi significativa no grupo de desnutridos $(47 \%)$, concordando com outros trabalhos desenvolvidos no pais $23,24,25$ os quais mostram os distúrbios intestinais ocupando lugar de destaque em freqüência de enfermidades infecciosas em crianças, com comprometimento do estado nutricional.

$\mathrm{SABRA}^{2-4}$ afirma ser a sindrome diarréica uma das causas mais importantes de morbidade e mortalidade no período neonatal e na infância não só no Brasil como em inúmeros países do mundo; encontra-se relacionada a fatores sócio-econômicos alcançando taxas de mortalidade alarmantes no nordeste brasileiro. 


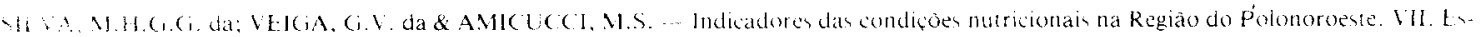

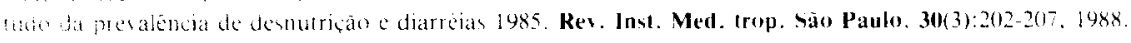

SiculEM ${ }^{2 \vdots}$ em São Paulo, observou não $\therefore$ maior freqüencia de distúrbios gastrointestiais em crianças desnutridas, como também, maitr ocorrência de distúrbios graves do aparetho gastrointestinal nestas crianças, com maior niimero de intercorrências e internaçôes hospitialares.

Os resultados obtidos no nosso estudo sobre prevalência de desnutrição e frequência de diarréias em Cáceres - Mato Grosso, mostraran resultados concordantes com outros já realaidos ctm crianças de outras regiões, o que parice fortalecer o elo estabelecido entre o estado nur onal e os processos infecciosos.

\section{SUMMARY}

Indicators of nutritional status in the Polonoroeste area. VII - Prevalence of ma!nutrition and diarrhea in $\mathbf{1 9 8 5}$

Vurtitional and anthropometric study of 4is wititen between 3 and 72 months of age 3.: in uball Caceres, Polonoroeste area, MT. Diarrhea was also studied. Nutrition was evaluatcd according the criteria of GOMEZ and W ATERLOW. According to GOMEZ critoria $33 \%$ of the children were undernourished and $36 \%$ of them it preceeded the lime of inquire. Forty seven percent of the undernourished children referred diarrhea and the correlation between diarrhea and undernutrition was significant.

\section{REFERÊNCIAS BIBLIOGRÁFICAS}

1. ATAL.AH S., E.; BUSTOS M., P. \& GOMEZ A., H - Desnutrición infantil: costo social por patologia respiratoria y digestiva. Arch. lat.-amer. Nutr., 33: 395 408,1983

2. INSEIMO, MA.C; ALENCAR, S.F. \& BURINI, R.C. -... Avaliaçào do estado nutricional Métodos bioqumicon. Rev, Soce bras. Nutr. Parent.. 2(4): 11-18. 1985

3. BAIISIA Fll HO, M. - Prevalência e estágios da desnutrição protéico calórica em crianças da cidade de São Paulo. São Paulo, 1976. (Tese de Doutoramento - Caculdade de Saude Pública da Universidade de São Paislor

- GRTA E ROMANL S A M BAHSTA IIHO V \& RQKHA, A.N.-... Desnutriçào recente, cronica e meressa em 9 localidades do estado de Pernambuco. Rev. Saúde públ. (S. Paulo), 15:211-220,1981.

$\therefore$ CARVALHO, A.A. - O pediatra e a desnuirição proteico täbrica. In: NOBRE(jA, F.J. - Desnutriçao intra uterina e pós natal. Sào Paulo, Janamed. 1981. p. $55+57$.
6. D'ANS, C.D. \& DRICOT, J.M. - Metodologia antropométrica do diagnóstico nutricional. Rev. Saúde públ. (S. Paulo), 16: 42-53, 1982.

7. FARHAT, C.K. - Desnutrição protéico calórica e in fecçào. In: NÓBREGA, F.J. - Desnutrição intra uterina e pós natal. São Paulo, Panamed, 1981. p. 287. 293.

8. GALLO, A.B. - Interación entre infección y nutrición. Rev. Chil. Pediat., 54: 63-66, 1983.

9. GOLDENBERG, P. - Organização social e desnutricào em famílias de baixa renda no Município de São Paulo. São Paulo, 1982. (Tese de Doutoramento - Fa. culdade de Saúde Pública da Universidade de Sào Paulo)

10. GOMEZ, F.; RAMOS GALVAN, R.; FRENK, S.; CRAVIOTO MUÑOZ, J.: CHÁVEZ, R. \& VAZ QUEZ, 1. - Mortality in second and thrd degree malnutrition. J. trop. Pediat., 2: 77-83, 1956.

11. GORDON, J.E. - Synergism of malnutrition and in fectious disease. Vutrition in preventive medicise. Ge neva, World Health Organization, 1976. D. 197-209.

12. HAMHL, P.V.V.; DRIZO T.A., JOHNSON C.L. REED, R.B.; ROCHE, A.S, \& MOCRE, Y.M. Phisical growth: National Center for Health Statistic percentiles. Amer. J. clin. Nutr. 32: 607-629, 1979.

13. JEL LIEFE, D. B. - Evaluacion del estado de nutrición de la comunidad. Ginebra, Organización Mundial de la Salud, 1966. (Serie de monogratias, 53)

14. MEIRELLES, S.M.P. \& SANCHEZ, O - Adaplaçà do "Desenho Anostral" elaborado para a pesquisa en: Saúde Pública no Polonoroeste - MT. Rev. Univ. Fed. Mato Grosso, 4(3):96-108, 198.4

15. MONTKIRO, (.A. -- Os determinantes da desnutri çào infantil no Vale do Ribeira. Cadern. pesq. (Fund. (arlos Chagas), (29): 57-74, 1975.

16. MONTEIRO, C.A. - Avaliação do estado nutricional na idade pré-escolar em áreas de baixa renda do Estado de São Paulo. Sào Paulo, 1982. (Tese de Livre Docência - Faculdade de Saúde Pública)

17. MASUDA, A. \& ROCHA, G.M. -- Desnutrição protéico calórica e infeç̧òes na infância. Medicina (Ribeirāo Pretol, 15: 107-112, 1982.

18. MATA, L..J. - Malnutrition infection interations in the tropics. Amer. J. trop. Med. Hig., 24: 564-574, 1975.

19. PUFFER, R.R. \& SERRANO, C.V. - Características de la mortalidad en la niñez. Washington, Organizacion Panamericana de la Salud, 1973. (Publicación (ientifica No. 262)

20. NOBREGA, F.J. -- Desnutriçao intra uterina e pósnatal. São Paulo, Panamed, 1981.

21. NEUMANN, C.G.; JELLIFFE, D.B. \& JFLLIFFE, E.F.P. - Interation of nutrition and infection. Clin. Pediat., 17: 807-812, 1978.

22. SCRIMSHAW, N.S. TAYLOR, C.E. \& GORDON J.E - Nutrición e infecciones: su acción recíproca. Gi nebra, Organización Mundial de la Salud, 1970. (Seric de monogratias, 57)

23. SICiULEN, D.M. - Condiçōes de saúde e nutrição de lactentes no municipio de São Paulo. São Paulo, 1981. (Tese de Doutoramento - Escola Paulista de Medi(ina)

24. SABRA, A. - Diarréias agudas na infância. J. bras. Med., 45: 17-35, 1983. 
SH VA, M.H.G.C, da; VEIGA. G.V. da \& AMICUCCI, M.S. - Indicadores das condicoes nutricionais na Regiào do Polonoroeste. Vll, Estudo da ptcralência de desilutricàu è diarretas 1485. Rer. Inst. Med. trop. São Paulo, 30(3):202-207, 1988.

25. TEIXEIRA D.E.A.; REZENDE, A.L.S.; MIRAN DA, I.A. \& FONTENELLE, V.L.C. - Frequência da morbidade em crianças menores de 3 anos em diferentes estados nutricionais. Hiléia méd., 5: 5-15, 1983.

26. VIACAVA, F.; FIGUEIREDO, C.M.P. \& OLIVEIRA, W.A. - A desnutrição no Brasil: uma análise do esiudo nacional da despesa familiar (IBGE 74/75) para o Nordeste, Estado de Sào Paulo e Estado do Rio de Janeiro. Petrópolis, Vozes, 1983. Cap. 2, p. 111.

27. WATERLOW, J.C. - Classification and definition protein energy malnutrition: nutrition in preventive medicine. Geneva, World Health Organization, 1976. p. 530-555. 